\title{
Cosmic string loop distribution on all length scales and at any redshift
}

\author{
Larissa Lorenz \\ E-mail: larissa.lorenz@uclouvain.be \\ Institute of Mathematics and Physics, Centre for Cosmology, Particle Physics and \\ Phenomenology, \\ Louvain University, 2 Chemin du Cyclotron, 1348 Louvain-la-Neuve, Belgium
}

\section{Christophe Ringeval}

E-mail: christophe.ringeval@uclouvain.be

Institute of Mathematics and Physics, Centre for Cosmology, Particle Physics and Phenomenology,

Louvain University, 2 Chemin du Cyclotron, 1348 Louvain-la-Neuve, Belgium

\section{Mairi Sakellariadou}

E-mail: mairi.sakellariadou@kcl.ac.uk

Department of Physics, King's College, University of London, Strand, London WC2R 2LS, United Kingdom

\begin{abstract}
We analytically derive the expected number density distribution of Nambu-Goto cosmic string loops at any redshift soon after the time of string formation to today. Our approach is based on the Polchinski-Rocha model of loop formation from long strings which we adjust to fit numerical simulations and complement by a phenomenological modelling of gravitational backreaction. Cosmological evolution drives the loop distribution towards scaling on all length scales in both the radiation and matter era. Memory of any reasonable initial loop distribution in the radiation era is shown to be erased well before Big Bang Nucleosynthesis. In the matter era, the loop distribution reaches full scaling, up to some residual loops from the radiation era which may be present for extremely low string tension. Finally, the number density of loops below the gravitational cutoff is shown to be scale independent, proportional to a negative power of the string tension and insensitive to the details of the backreaction modelling. As an application, we show that the energy density parameter of loops today cannot exceed $10^{-5}$ for currently allowed string tension values, while the loop number density cannot be less than $10^{-6}$ per $\mathrm{Mpc}^{3}$. Our result should provide a more robust basis for studying the cosmological consequences of cosmic string loops.
\end{abstract}

PACS numbers: 98.80.Cq, 98.70.Vc 


\section{Introduction}

It was shown by Kibble that cosmic strings are a natural outcome of cosmological phase transitions [1, 2, 3, 4], and their possible presence in our Universe has been extensively studied ever since. Cosmic strings come in the form of line-like topological defects produced by the spontaneous breakdown of some symmetry [5, 6, 7], but can also be stretched superstrings generated at the end of brane inflation [8, 9, 10, 11, 12, 13, 14, 15]. Once formed, cosmic strings evolve and very rapidly reach their so-called scaling regime in which the energy density $\rho_{\infty}$ of super-horizon sized strings behaves as radiation in the radiation era, or matter in the matter era [16, 17, 18, 19]. Hence, for these long strings it holds that $\rho_{\infty} \propto 1 / d_{\mathrm{h}}^{2}$, where $d_{\mathrm{h}}$ stands for the distance to the horizon $\left(d_{\mathrm{h}} \propto t\right.$, the cosmic time). This scaling ensures that long strings never dominate the energy density of the Universe at late times and remain compatible with cosmological observations provided their energy density per unit length $U$ is small enough. The current Cosmic Microwave Background (CMB) data limits the string contribution to at most $10 \%$ on the angular scales observed by the Wilkinson Microwave Anisotropies Probe (WMAP) satellite [20], i.e. $G U<7 \times 10^{-7}$ for Abelian vortices, $G$ being the Newton constant [21]. However, being present all along the Universe's history, cosmic strings may be the dominant source of CMB non-gaussianities and are expected to dominate over inflationary perturbations at small angular scales [22, 23, 24, 25, 26, 27, 28, 29, 30].

The existence of a scaling regime for Nambu-Goto (NG) string networks requires

the incessant formation of loops from long cosmic strings. Loops are produced at string autocommutation and intersection events, thereby evacuating the excess energy density from long strings. In the standard picture, the produced loops shrink and disappear by gravitational wave emission, which could provide additional observational evidence for cosmic strings [31, 32, 33, 34, 35. Note, however, that Abelian Higgs string networks preferentially reach scaling by boson radiation rather than loop formation, at least during their numerically probed evolution [36, 37, 38].

The expected cosmological distribution of loops has been subject to intense debate since the development of the original "one scale" model by Kibble [1]. In this model, at any time loops are formed with a size given by the typical distance separating long strings, i.e. a fraction of the distance to the horizon $d_{\mathrm{h}}$. NG numerical simulations in Friedmann-Lemaittre-Robertson-Walker (FLRW) spacetime have shown, however, that most loops are formed at much smaller size, suggesting the existence of tiny correlation lengths in cosmic string networks [17, 19, 39]. These simulations are nevertheless limited in time and cannot exceed a redshift range typically of order $10^{2}$. Comparing this to the $10^{18}$ change in redshift expected at Big Bang Nucleosynthesis (BBN) [provided strings are formed at the Grand Unified Theory (GUT) energy scale], it is crucial for simulations to disentangle transient effects from the ones lasting over cosmological timescales.

As shown in Refs. [40, 41, many of the small loops observed in Nambu-Goto simulations indeed come from a transient effect associated with the relaxation of the initial string network towards its stable configuration. One must not expect these loops 
to remain present after a long enough evolution. However, in addition to transient loops, Ref. [41] was the first to exhibit a sub-population of loops in scaling which constitute the cosmological attractor, a result recovered in Refs. [42, 43, 44], albeit with some differences in the distribution. The energy density distribution of loops in scaling follows an evolution similar to long strings, i.e. it behaves as radiation in the radiation era, and as matter in the matter era. In this regime, the loop number density distribution has a power law shape: it is "scale-free" in the sense that loops of all size are present at any time. These results can be explained if the dominant mechanism of string evolution also applies to loops: they mainly loose energy by in turn emitting other loops. More quantitatively, denoting by $\alpha=\ell / d_{\mathrm{h}}$ the size of a loop in units of $d_{\mathrm{h}}$, the loop number density $n(\alpha, t)$ and energy density $\rho_{\circ}(\alpha, t)$ in scaling read [41]

$$
\frac{\mathrm{d} n}{\mathrm{~d} \alpha}=\frac{\mathcal{S}(\alpha)}{\alpha d_{\mathrm{h}}^{3}}, \quad \frac{\mathrm{d} \rho_{\circ}}{\mathrm{d} \alpha}=U \frac{\mathcal{S}(\alpha)}{d_{\mathrm{h}}^{2}},
$$

where the "scaling function" $\mathcal{S}(\alpha)$ is well fitted, in the radiation and matter era, by the power laws $\mathcal{S}(\alpha)=C_{\circ} \alpha^{-p}$ with [41]

$$
\left\{\left.\begin{array}{c}
p=1.41_{-0.07}^{+0.08} \\
C_{\circ}=0.09_{+0.03}^{-0.03}
\end{array}\right|_{\mathrm{mat}}, \quad\left\{\left.\begin{array}{r}
p=1.60_{-0.15}^{+0.21} \\
C_{\circ}=0.21_{+0.13}^{-0.12}
\end{array}\right|_{\mathrm{rad}} .\right.\right.
$$

Note that the energy density of loops evolves as does the one of long strings, $\rho_{\circ} \propto 1 / d_{\mathrm{h}}^{2}$.

NG numerical simulations only account for string dynamics and intercommutation events, and in particular they know nothing about the impact of the loops' gravitational wave emission. In a realistic physical situation, a loop of size $\ell$ emitting gravitational waves is expected to decay within a time roughly given by $t_{\mathrm{d}}=\ell /(\Gamma G U)$, where $\Gamma$ is a numerical coefficient depending on the string structure, which for typical loops is of the order of $\Gamma \simeq 10^{2}$ [45, 31]. As a result, for small loops with $\alpha<\alpha_{\mathrm{d}} \propto \Gamma G U$, gravitational wave emission should overcome loop emission as the dominant energy loss mechanism. The (numerically found) scaling law of Eq. (2) can therefore no longer be used in the regime $\alpha<\alpha_{\mathrm{d}}$, and the loop distribution at those length scales must be understood further analytically, taking into account the emission of gravitational waves.

Another phenomenon associated with gravitational radiation is the so-called backreaction effect which applies to both loops and long strings. The emission of gravitational waves renders the strings smoother and smoother on the smallest length scales, hence preventing self-intersection over a very small distance [46, 47]. Therefore, long strings and loops cannot produce infinitely small loops: gravitational backreaction cuts off loop production below a certain scale. The typical length at which this happens was estimated to be of order $\ell_{\mathrm{c}} \propto(G U)^{1+2 \chi} t$, where $\chi>0$. When translated into the variable $\alpha$ and compared to the scale of gravitational decay $\alpha_{\mathrm{d}} \propto \Gamma G U$ introduced above, this corresponds to a constant value of $\alpha_{\mathrm{c}}<\alpha_{\mathrm{d}}$ [48, 49, 50].

The goal of this paper is to establish, both in the radiation and the matter dominated eras, a phenomenological description of the string loop number density evolution which includes the three above-mentioned effects: loop formation, decay by gravitational wave emission and the impact of gravitational backreaction. Various 
analytical models have been proposed so far to describe the evolution of a cosmic string network [51, 52, 53, 54]. Each of these models starts from a different set of assumptions and consequently, their results may differ. Our objective here is to describe the cosmic string loop distribution in the well-motivated situation where it coexists with a long string network in its scaling regime. Long strings indeed reach their cosmological attractor after a redshift range which does not exceed an order of magnitude [30]. For this reason, we use the Polchinski-Rocha (PR) approach of Refs. [55, 56] in which loops are formed from tangent vector correlations along long strings in scaling. Let us mention that a non-scaling loop production function has recently been introduced in Refs. [57, 58] to explain the existence of loops associated with the initial conditions. As discussed below (see Sec. 3.1), our approach accommodates the existence of such loops without invoking a non-scaling loop production function.

The PR model predictions are in good agreement with the correlators measured in Abelian Higgs simulations [38] and also with the NG loop distribution of Eq. (2) [59], up to the overall normalisation. This agreement may appear surprising since simulations include many additional effects on top of loop formation from long strings, e.g. loop formation from loops, loop fragmentation and loop reconnection leading to daughter loops of a different size than the parent ones, all of them being non-negligible [18]. The physical interpretation is that these additional mechanisms preserve the functional form of the analytical PR loop production function (which is derived for the long strings) and amount to a renormalization of its amplitude. The PR production function can thus be considered as a robust physical feature against these effects, and for this reason, we take it as an ansatz for the total loop production function yet to be established. However, we can no longer fix its unknown coefficients analytically by using the long string scaling properties since we want to extend its use beyond the "long strings only" picture. We can, however, choose these coefficients to match the numerical results of Eq. (2), in the appropriate range of length scales.

Below, we extend the method originally introduced by Rocha in Ref. [59] along the three following directions. Firstly, we introduce a change in behaviour of the loop production function below the gravitational backreaction length scale. The true shape of the loop production function in this regime being unknown, we consider various motivated possibilities and show that the final loop number density distribution remains unchanged. Secondly, we do not a priori neglect the transient solutions, which allows us to discuss the effect of the initial loop number density distribution at string formation time. This is the subject of Sec. 2. In Sec. 3, we discuss how the distribution evolves with redshift through the radiation and matter eras, as well as through the transition. We find that all loops rapidly reach the so-called "full-scaling regime", in which their number density assumes the universal form plotted in Fig. 2. We furthermore derive the loops' energy density parameter and the loop number density today before presenting our conclusions in Sec. 4 . 


\section{Cosmological attractor}

Borrowing notation from Ref. [59], the distance to the horizon in terms of cosmic time $t$ is expressed as

$$
d_{\mathrm{h}}(t)=\frac{t}{1-\nu},
$$

where the scale factor $a(t) \propto t^{\nu}$, with $\nu=1 / 2$ in the radiation era and $\nu=2 / 3$ in the matter era. Moreover, the cosmic time $t$ can be expressed in terms of redshift $z$, of the Hubble parameter and of the density parameters today $\left(H_{0}, \Omega_{\mathrm{r}_{0}}\right.$ and $\Omega_{\mathrm{m}_{0}}$, respectively) as

$$
t_{\mathrm{rad}}(z) \simeq \frac{1}{2 H_{0} \sqrt{\Omega_{\mathrm{r}_{0}}}} \frac{1}{(1+z)^{2}},
$$

in the radiation era and as

$$
t_{\mathrm{mat}}(z) \simeq \frac{2}{3 H_{0} \sqrt{\Omega_{\mathrm{m} 0}}} \frac{1}{(1+z)^{3 / 2}},
$$

in the matter era. The above expressions constitute a very good approximation of the currently favoured $\Lambda \mathrm{CDM}$ (Lambda Cold Dark Matter) model [60] up to the smooth transition from the radiation to the matter era, and before the cosmological constant domination sets in (i.e. for redshifts $z>2$ ). Note that, from Eqs. (44) and (5), the instantaneous transition between the two eras occurs at

$$
z_{*}=\frac{9}{16} \frac{\Omega_{\mathrm{m}_{0}}}{\Omega_{\mathrm{r}_{0}}}-1=\frac{9}{16}\left(1+z_{\mathrm{eq}}\right)-1,
$$

where $z_{\text {eq }}$ stands for the redshift of equality between the radiation and matter energy densities.

\subsection{Evolution equation}

Denoting by $n(\ell, t)$ the number density distribution of cosmic string loops of size $\ell$ at cosmic time $t$, we can write in the Eulerian description

$$
\frac{\mathrm{d}}{\mathrm{d} t}\left(a^{3} \frac{\mathrm{d} n}{\mathrm{~d} \ell}\right)=a^{3} \mathcal{P}(\ell, t),
$$

where $\mathcal{P}(\ell, t)$ is the loop production function, i.e. the number density distribution of loops of size $\ell$ produced per unit of time at $t$. In the Lagrangian description, due to gravitational radiation, a loop of size $\ell(t)$ shrinks at a constant rate such that

$$
\frac{\mathrm{d} \ell}{\mathrm{d} t}=-\gamma_{\mathrm{d}},
$$

which we take as the definition of $\gamma_{\mathrm{d}}$. According to the discussion of Sec. 1, and using Eqs. (3) and (8)), we have

$$
\gamma_{\mathrm{d}}=\frac{\alpha_{\mathrm{d}}}{1-\nu} \simeq \Gamma G U .
$$

Combining Eqs. (77) and (8), one gets [59]

$$
\frac{\partial}{\partial t}\left(a^{3} \frac{\mathrm{d} n}{\mathrm{~d} \ell}\right)-\gamma_{\mathrm{d}} \frac{\partial}{\partial \ell}\left(a^{3} \frac{\mathrm{d} n}{\mathrm{~d} \ell}\right)=a^{3} \mathcal{P}(\ell, t) .
$$


As suggested by the form of the scaling distribution in Eq. (11), it is more convenient to express the loop sizes $\ell$ in units of the horizon $d_{\mathrm{h}}$. In fact, since we shall be interested in the radiation matter transition, it is even more convenient to work in units of cosmic time $t$. For this purpose, let us define a new variable $\gamma$ and a new function $\mathcal{F}$, as

$$
\gamma(\ell, t) \equiv \frac{\ell}{t}, \quad \mathcal{F}(\gamma, t) \equiv \frac{\mathrm{d} n}{\mathrm{~d} \ell},
$$

respectively, in terms of which Eq. (10) reads

$$
t \frac{\partial\left(a^{3} \mathcal{F}\right)}{\partial t}-\left(\gamma+\gamma_{\mathrm{d}}\right) \frac{\partial\left(a^{3} \mathcal{F}\right)}{\partial \gamma}=a^{3} t \mathcal{P}(\gamma, t) .
$$

Evidently, the time variable $t$ ranges from the initial time of string network formation $\left(t_{\text {ini }}\right)$ to today $\left(t_{0}\right)$, while $0 \leq \gamma \leq \gamma_{\max }$, where $\gamma_{\max }$ corresponds to $l_{\max }$, the size of the largest (horizon-sized) loops, which will be discussed below.

\subsection{Loop production function}

The scaling law of Eq. (1) implies that, once the scaling regime is reached, $t^{4} \mathcal{F}(\gamma, t)$ should be a function of $\gamma$ only. From Eq. (12), we expect the same to happen for $t^{5} \mathcal{P}(\gamma, t)$. Following Refs. [55, 56, 59], we moreover assume that this function is a power law, namely

$$
t^{5} \mathcal{P}(\gamma, t)=c \gamma^{2 \chi-3},
$$

where $c$ and $\chi$ are two parameters that will be fixed to fit Eq. (2). However, according to our discussion of Sec. 1, the above expression is only valid for a range of $\gamma$ values where gravitational backreaction effects can be neglected. Hence, Eq. (13) holds for values of $\gamma$ greater than

$$
\gamma_{\mathrm{c}} \equiv \frac{\alpha_{\mathrm{c}}}{1-\nu} \simeq \Upsilon(G U)^{1+2 \chi},
$$

where $\Upsilon$ is a number $\mathcal{O}(10)$ [50]. The parameter $\chi$ in this expression is expected to be the same as in Eq. (13), given that the gravitational backreaction length scale is precisely derived from the same two-point correlators used in the PR model to obtain Eq. (13) (see Ref. [50]). In our phenomenological approach, we consider $\gamma_{\mathrm{c}}$ as a free parameter below which the loop production function is given by a different power law,

$$
t^{5} \mathcal{P}\left(\gamma<\gamma_{\mathrm{c}}, t\right)=c_{\mathrm{c}} \gamma^{2 \chi_{\mathrm{c}}-3} .
$$

The parameters $c_{\mathrm{c}}$ and $\chi_{\mathrm{c}}$ are such that the loop production function is continuous across $\gamma=\gamma_{\mathrm{c}}$, which leads to

$$
c_{\mathrm{c}}=c \gamma_{\mathrm{c}}^{2\left(\chi-\chi_{\mathrm{c}}\right)} \text {. }
$$

Moreover, since gravitational backreaction smooths the strings on the smallest length scales, less loops should be produced in the small $\gamma$ limit, and one may naively expect the production function $\mathcal{P}(\gamma, t)$ to vanish for $\gamma=0$. In fact, by incorporating backreaction effects into the long string structure, it has been shown in Ref. [50] that, for $\gamma \simeq \gamma_{\mathrm{c}}$, 
Eq. (13) has a power law exponent renormalised to $\chi_{\mathrm{br}} \simeq 1$. This gives an extreme lower limit for $\chi_{\mathrm{c}}$,

$$
\chi_{\mathrm{c}} \geq 1,
$$

as backreaction cannot smooth the string structure less than that. From Eq. (15) we therefore see that the loop production function can be divergent in the $\gamma \rightarrow 0$ limit for $1<\chi_{\mathrm{c}}<3 / 2$. However, being its first integral, the loop number density $\mathcal{F}(\gamma, t)$ will remain finite. Only in the limiting case $\chi_{\mathrm{c}}=1$ would we find a logarithmic $\gamma \rightarrow 0$ divergence for $\mathcal{F}(\gamma, t)$ (see below), which nevertheless leads to a finite energy density (which is $\propto U \ell \mathcal{F}$ ). This extreme situation corresponds to the production of an infinite number of infinitely small loops but with finite energy density. On the opposite, if gravitational backreaction was infinitely effective, no loops at all would be produced with $\gamma<\gamma_{\mathrm{c}}$, corresponding to the limit $\chi_{\mathrm{c}} \rightarrow \infty$. Certainly the true physical situation lies somewhere between these two extremes [51]. In the following, we show that the final loop number density distribution is (almost) insensitive to the chosen value of $\chi_{\mathrm{c}}$, and thus to the details of the backreaction smoothing process. Only the cutoff scale $\gamma_{c}$ enters into the expression for $t^{4} \mathcal{F}(\gamma, t)$ in its scaling regime. A sketch of the loop production function $t^{5} \mathcal{P}(\gamma, t)$ as a function of $\gamma$ is drawn in Fig. 1.

With Eqs. (13) and (15) we now have at our disposal a continuous expression for the loop production function for all $\gamma \geq 0$. As mentioned earlier, however, there exists an upper bound $\gamma_{\max }$ on the accessible range of $\gamma$ : any loop with $\gamma>1 /(1-\nu)$ is of superhorizon size and hence indistinguishable from a long string. Hence, its contribution has already been included in the loop production function. In fact, in a cosmological string network, there does exist a small population of loops with size comparable to $d_{\mathrm{h}}$. We shall refer to them as "Kibble's loops", and they are not described by our current approach [41, 42]. Due to Hubble damping, their properties are in all aspects comparable to those of long strings, and they essentially decay by loop emission rather than gravitational radiation. As for long strings, we consider their net effect included in the loop production function. The typical size of these loops is given by the distance between two super-horizon sized strings in scaling, which is

$$
\gamma_{\infty}=\frac{1}{1-\nu}\left(\frac{U}{\rho_{\infty} d_{\mathrm{h}}^{2}}\right)^{1 / 2} .
$$

From the values of $\rho_{\infty}$ given in Ref. [41], one gets $\gamma_{\infty} \simeq 0.32$ in the radiation era and $\gamma_{\infty} \simeq 0.56$ in the matter era. As we shall see later, our model predicts no loop larger than these values. However, in a realistic situation one should bare in mind that typically a few Kibble's loops are present.

Finally, it has been shown in Ref. 61] that kinks propagating along the strings can also smooth the tangent vector correlator used in the PR model of loop formation. This amounts to setting $\chi=1 / 2$ in Eq. (13) for the $\gamma$ values at which this effect appears, i.e. it would require the addition of another piecewise domain in the definition of the loop production function. Although possible, we have chosen not to include kink smoothing in the following considerations since this effect is expected to be significant 


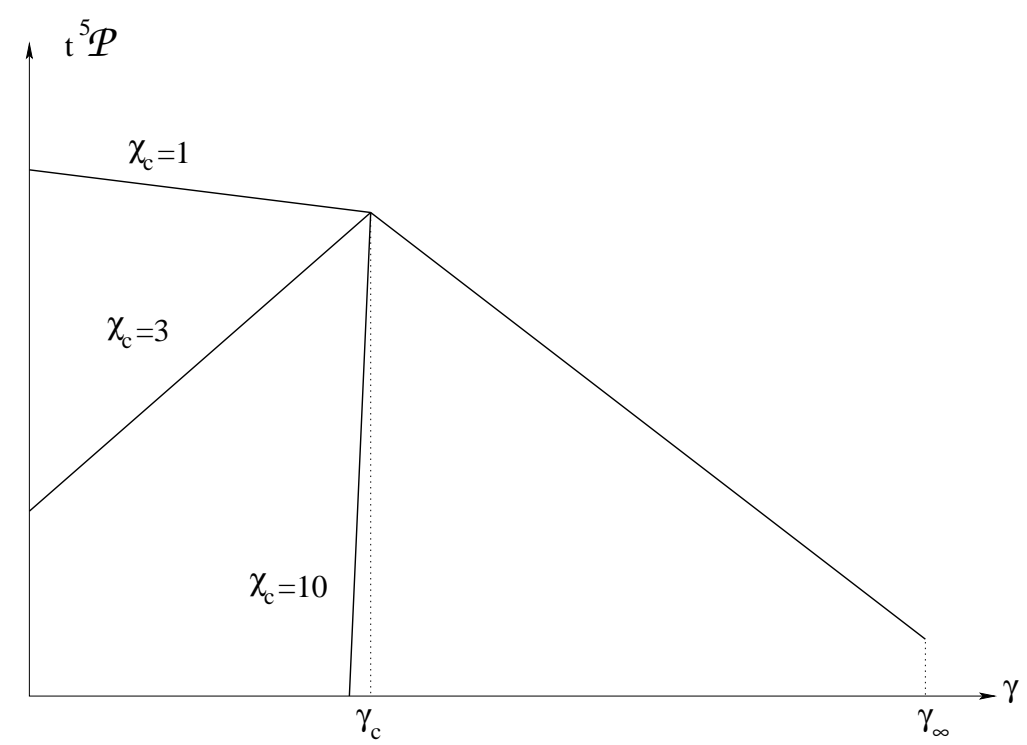

Figure 1. Sketch of the loop production function $t^{5} \mathcal{P}(\gamma, t)$ as a function of $\gamma$ in logarithmic units. The gravitational backreaction scale is located at $\gamma=\gamma_{\mathrm{c}}$.

in a domain $\gamma<\gamma_{\mathrm{k}}(t)$, where $\gamma_{\mathrm{k}}(t)$ is a decreasing function of time [61]. As a result, the effect is transient and should no longer be observable when $\gamma_{\mathrm{k}}(t)$ hits the gravitational backreaction length scale $\gamma_{c}$.

\subsection{Evolution from an initial distribution}

We now assume the loop number density distribution is known at some initial time $t_{\text {ini }}$, when it is given by

$$
\mathcal{N}_{\text {ini }}(\ell) \equiv \frac{\mathrm{d} n}{\mathrm{~d} \ell}\left(\ell, t_{\text {ini }}\right) .
$$

For instance, the initial loop distribution can be obtained by examining the statistical properties of the Higgs field phases just after the phase transition responsible for string formation [62, 63, 64. However, since the loop production function of Eq. (13) assumes a scaling form, $\mathcal{N}_{\text {ini }}(\ell)$ should be understood as the loop distribution expected at least just after the scaling of long strings takes place. In the following, we solve Eq. (12), with the two-fold loop production function of Eqs. (13) and (15), starting from a given $\mathcal{N}_{\text {ini }}$ at $t=t_{\text {ini }}$.

Using new variables $w$ and $s$ defined from

$$
w=\ln t, \quad s=\ln t+\ln \left(\gamma+\gamma_{\mathrm{d}}\right),
$$

as well as Eq. (13) for the loop production function, Eq. (12) simplifies to

$$
\frac{\partial}{\partial w}\left(a^{3} \mathcal{F}\right)=c a^{3} \mathrm{e}^{-4 w}\left(\mathrm{e}^{s-w}-\gamma_{\mathrm{d}}\right)^{2 \chi-3}
$$

for $\gamma>\gamma_{\mathrm{c}}$. The same expression holds for $\gamma<\gamma_{\mathrm{c}}$ up to the replacement $c \rightarrow c_{\mathrm{c}}$ and $\chi \rightarrow \chi_{\mathrm{c}}$. Assuming $\gamma_{\mathrm{d}}$ constant and $a \propto t^{\nu}$, this equation is readily integrated in terms 
Cosmic string loop distribution on all length scales and at any redshift

of the Gauss hypergeometric function. For $\gamma>\gamma_{c}$, the solution of Eq. (21) reads

$$
\mathcal{F}(\gamma, t)=\frac{c}{\mu} \frac{\left(\gamma+\gamma_{\mathrm{d}}\right)^{2 \chi-3}}{t^{4}}{ }_{2} \mathrm{~F}_{1}\left(3-2 \chi, \mu ; \mu+1 ; \frac{\gamma_{\mathrm{d}}}{\gamma+\gamma_{\mathrm{d}}}\right)+\frac{\mathcal{I}\left(\gamma t+\gamma_{\mathrm{d}} t\right)}{a^{3}},
$$

where $\mathcal{I}(x)$ is an arbitrary function (still to be determined) and

$$
\mu \equiv 3 \nu-2 \chi-1 \text {. }
$$

For $\gamma<\gamma_{c}$, one obtains exactly the same expression up to the replacement of $c$ and $\chi$ into $c_{\mathrm{c}}$ and $\chi_{\mathrm{c}}$ [hence, $\mu_{\mathrm{c}}$ is defined as in Eq. (23) by replacing $\chi \rightarrow \chi_{\mathrm{c}}$ ]. The unknown integration function in this regime is denoted by $\mathcal{I}_{\mathrm{c}}(x)$. In order to simplify the notations, we also define

$$
f(x) \equiv{ }_{2} \mathrm{~F}_{1}(3-2 \chi, \mu ; \mu+1 ; x), \quad f_{\mathrm{c}}(x) \equiv{ }_{2} \mathrm{~F}_{1}\left(3-2 \chi_{\mathrm{c}}, \mu_{\mathrm{c}} ; \mu_{\mathrm{c}}+1 ; x\right),
$$

and the rescaled constants

$$
C \equiv \frac{c}{\mu}, \quad C_{\mathrm{c}} \equiv \frac{c_{\mathrm{c}}}{\mu_{\mathrm{c}}}=\frac{\mu}{\mu_{\mathrm{c}}} \gamma_{\mathrm{c}}^{2\left(\chi-\chi_{\mathrm{c}}\right)} C .
$$

The loop number density distribution in its two respective domains then reads

$$
\begin{aligned}
& \mathcal{F}\left(\gamma \geq \gamma_{\mathrm{c}}, t\right)=C \frac{\left(\gamma+\gamma_{\mathrm{d}}\right)^{2 \chi-3}}{t^{4}} f\left(\frac{\gamma_{\mathrm{d}}}{\gamma+\gamma_{\mathrm{d}}}\right)+\frac{\mathcal{I}\left[\left(\gamma+\gamma_{\mathrm{d}}\right) t\right]}{a^{3}}, \\
& \mathcal{F}\left(\gamma<\gamma_{\mathrm{c}}, t\right)=C_{\mathrm{c}} \frac{\left(\gamma+\gamma_{\mathrm{d}}\right)^{2 \chi_{\mathrm{c}}-3}}{t^{4}} f_{\mathrm{c}}\left(\frac{\gamma_{\mathrm{d}}}{\gamma+\gamma_{\mathrm{d}}}\right)+\frac{\mathcal{I}_{\mathrm{c}}\left[\left(\gamma+\gamma_{\mathrm{d}}\right) t\right]}{a^{3}} .
\end{aligned}
$$

Continuity of $\mathcal{F}(\gamma, t)$ across $\gamma=\gamma_{\mathrm{c}}$ at all times imposes that the unknown functions $\mathcal{I}(x)$ and $\mathcal{I}_{\mathrm{c}}(x)$ are not independent. From Eqs. (26) and (27) one then obtains the relation

$$
\mathcal{I}_{\mathrm{c}}(x)=\mathcal{I}(x)+K \frac{\left(\gamma_{\mathrm{d}}+\gamma_{\mathrm{c}}\right)^{4}}{x^{4}}\left[a\left(\frac{x}{\gamma_{\mathrm{c}}+\gamma_{\mathrm{d}}}\right)\right]^{3},
$$

where $K$ is a constant given by

$$
K=C\left(\gamma_{\mathrm{c}}+\gamma_{\mathrm{d}}\right)^{2 \chi-3} f\left(\frac{\gamma_{\mathrm{d}}}{\gamma_{\mathrm{c}}+\gamma_{\mathrm{d}}}\right)-C_{\mathrm{c}}\left(\gamma_{\mathrm{c}}+\gamma_{\mathrm{d}}\right)^{2 \chi_{\mathrm{c}}-3} f_{\mathrm{c}}\left(\frac{\gamma_{\mathrm{d}}}{\gamma_{\mathrm{c}}+\gamma_{\mathrm{d}}}\right) .
$$

Given $\mathcal{N}_{\text {ini }}(\ell)$, we can now uniquely determine the two unknown functions. At $t=t_{\text {ini }}$, defining

$$
x_{\text {ini }} \equiv\left(\gamma_{\mathrm{c}}+\gamma_{\mathrm{d}}\right) t_{\mathrm{ini}}
$$

one can use Eq. (26) to get $\mathcal{I}(x)$ for all $x>x_{\text {ini: }}$ :

$$
\mathcal{I}(x)=a_{\text {ini }}^{3} \mathcal{N}_{\text {ini }}\left(x-\gamma_{\mathrm{d}} t_{\text {ini }}\right)-C \frac{a_{\text {ini }}^{3}}{t_{\text {ini }}^{4}}\left(\frac{x}{t_{\text {ini }}}\right)^{2 \chi-3} f\left(\frac{\gamma_{\mathrm{d}}}{x} t_{\text {ini }}\right) .
$$

This expression also provides $\mathcal{I}_{\mathrm{c}}(x)$ for all $x>x_{\text {ini }}$ by using Eq. (28). Similarly, setting $t=t_{\text {ini }}$ into Eq. (27) uniquely determines $\mathcal{I}_{\mathrm{c}}(x)$ for all $x<x_{\text {ini }}$,

$$
\mathcal{I}_{\mathrm{c}}(x)=a_{\text {ini }}^{3} \mathcal{N}_{\text {ini }}\left(x-\gamma_{\mathrm{d}} t_{\text {ini }}\right)-C_{\mathrm{c}} \frac{a_{\text {ini }}^{3}}{t_{\text {ini }}^{4}}\left(\frac{x}{t_{\text {ini }}}\right)^{2 \chi_{\mathrm{c}}-3} f_{\mathrm{c}}\left(\frac{\gamma_{\mathrm{d}}}{x} t_{\text {ini }}\right),
$$


which now gives $\mathcal{I}\left(x<x_{\text {ini }}\right)$ from Eq. (28). After some algebra, plugging Eqs. (28) and (32) into Eq. (26) and (27), where the argument of the function $\mathcal{I}$ is now given by $\left(\gamma+\gamma_{\mathrm{d}}\right) t$, yields the final solution

$$
\begin{aligned}
& t^{4} \mathcal{F}\left(\gamma \geq \gamma_{\mathrm{c}}, t\right) \quad=\left(\frac{t}{t_{\text {ini }}}\right)^{4}\left(\frac{a_{\text {ini }}}{a}\right)^{3} t_{\text {ini }}^{4} \mathcal{N}_{\text {ini }}\left\{\left[\gamma+\gamma_{\mathrm{d}}\left(1-\frac{t_{\text {ini }}}{t}\right)\right] t\right\} \\
& +C\left(\gamma+\gamma_{\mathrm{d}}\right)^{2 \chi-3} f\left(\frac{\gamma_{\mathrm{d}}}{\gamma+\gamma_{\mathrm{d}}}\right) \\
& -C\left(\gamma+\gamma_{\mathrm{d}}\right)^{2 \chi-3}\left(\frac{t}{t_{\mathrm{ini}}}\right)^{2 \chi+1}\left(\frac{a_{\mathrm{ini}}}{a}\right)^{3} f\left(\frac{\gamma_{\mathrm{d}}}{\gamma+\gamma_{\mathrm{d}}} \frac{t_{\mathrm{ini}}}{t}\right), \\
& t^{4} \mathcal{F}\left(\gamma_{\tau} \leq \gamma<\gamma_{\mathrm{c}}, t\right)=\left(\frac{t}{t_{\text {ini }}}\right)^{4}\left(\frac{a_{\text {ini }}}{a}\right)^{3} t_{\text {ini }}^{4} \mathcal{N}_{\text {ini }}\left\{\left[\gamma+\gamma_{\mathrm{d}}\left(1-\frac{t_{\text {ini }}}{t}\right)\right] t\right\} \\
& +C_{\mathrm{c}}\left(\gamma+\gamma_{\mathrm{d}}\right)^{2 \chi_{\mathrm{c}}-3} f_{\mathrm{c}}\left(\frac{\gamma_{\mathrm{d}}}{\gamma+\gamma_{\mathrm{d}}}\right) \\
& -C\left(\gamma+\gamma_{\mathrm{d}}\right)^{2 \chi-3}\left(\frac{t}{t_{\text {ini }}}\right)^{2 \chi+1}\left(\frac{a_{\text {ini }}}{a}\right)^{3} f\left(\frac{\gamma_{\mathrm{d}}}{\gamma+\gamma_{\mathrm{d}}} \frac{t_{\text {ini }}}{t}\right) \\
& +K\left(\frac{\gamma_{\mathrm{c}}+\gamma_{\mathrm{d}}}{\gamma+\gamma_{\mathrm{d}}}\right)^{4}\left[\frac{a\left(\frac{\gamma+\gamma_{\mathrm{d}}}{\gamma_{\mathrm{c}}+\gamma_{\mathrm{d}}} t\right)}{a(t)}\right]^{3}, \\
& t^{4} \mathcal{F}\left(0<\gamma<\gamma_{\tau}, t\right)=\left(\frac{t}{t_{\text {ini }}}\right)^{4}\left(\frac{a_{\text {ini }}}{a}\right)^{3} t_{\text {ini }}^{4} \mathcal{N}_{\text {ini }}\left\{\left[\gamma+\gamma_{\mathrm{d}}\left(1-\frac{t_{\text {ini }}}{t}\right)\right] t\right\} \\
& +C_{\mathrm{c}}\left(\gamma+\gamma_{\mathrm{d}}\right)^{2 \chi_{\mathrm{c}}-3} f_{\mathrm{c}}\left(\frac{\gamma_{\mathrm{d}}}{\gamma+\gamma_{\mathrm{d}}}\right) \\
& -C_{\mathrm{c}}\left(\gamma+\gamma_{\mathrm{d}}\right)^{2 \chi_{\mathrm{c}}-3}\left(\frac{t}{t_{\mathrm{ini}}}\right)^{2 \chi_{\mathrm{c}}+1}\left(\frac{a_{\mathrm{ini}}}{a}\right)^{3} f_{\mathrm{c}}\left(\frac{\gamma_{\mathrm{d}}}{\gamma+\gamma_{\mathrm{d}}} \frac{t_{\mathrm{ini}}}{t}\right),
\end{aligned}
$$

where we have defined

$$
\gamma_{\tau}(t) \equiv\left(\gamma_{\mathrm{c}}+\gamma_{\mathrm{d}}\right) \frac{t_{\text {ini }}}{t}-\gamma_{\mathrm{d}}
$$

Let us stress that, although the solution is defined on three different domains, it is continuous by construction. The "middle domain" $\gamma \in\left[\gamma_{\tau}, \gamma_{\mathrm{c}}\right]$ owes its existence to two effects. Loops with a size $\ell<\gamma_{\mathrm{c}} t$ at time $t$ can either come from "on-site production" with a $\gamma<\gamma_{\mathrm{c}}$, or they may have started out earlier as larger loops, having $\gamma>\gamma_{\mathrm{c}}$, which then have shrunk into the domain $\gamma<\gamma_{\mathrm{c}}$ by gravitational radiation. Of course, the same mechanism applies to all loops in the initial distribution, as it can be seen from the argument of $\mathcal{N}_{\text {ini }}$ in Eq. (33) $)$, weighted by a dilution factor $a^{-3}$. The third domain $\gamma<\gamma_{\tau}$ corresponds to the virgin population of loops that started out with a $\gamma<\gamma_{\mathrm{c}}$ and which knows nothing about shrunk loops produced at $\gamma>\gamma_{\mathrm{c}}$. As one expects, these "non-contaminated" loops cannot exist indefinitely and this domain of the solution only exists transiently. From Eq. (34), we find that $\gamma_{\tau}\left(t_{\tau}\right)=0$ for

$$
\frac{t_{\tau}-t_{\mathrm{ini}}}{t_{\mathrm{ini}}}=\frac{\gamma_{\mathrm{c}}}{\gamma_{\mathrm{d}}} .
$$


Clearly, if $\gamma_{\mathrm{c}} \ll \gamma_{\mathrm{d}}$, this region exists only for a negligible duration after $t_{\mathrm{ini}}$. Even in the unlikely case of $\gamma_{\mathrm{c}}=\gamma_{\mathrm{d}}$, one finds $t_{\tau}=2 t_{\text {ini }}$, i.e. at most one Hubble time. In the following, we neglect this $\gamma$ domain, i.e. we shall use only the first two solutions given in Eq. (33) for the loop number density distribution.

\subsection{Asymptotic expansions}

We are now in a position to determine the unknown constants in Eq. (33) by matching them to the scaling solutions obtained in NG numerical simulations [41]. Since $\mathcal{N}_{\text {ini }}\left(\ell>d_{\mathrm{h} \mid \text { ini }}\right)=0$, for any fixed $\gamma$ there exists a time at which all terms involving the initial loop distribution $\mathcal{N}_{\text {ini }}$ in Eq. (33) vanish. This relaxation from the initial loop distribution towards the scaling regime will be discussed in detail in Sec. 3. Taking the limit $t \gg t_{\text {ini }}$ and restricting our attention to the domain probed by numerical simulations, i.e. $\gamma \gg \gamma_{\mathrm{d}}$, one gets

$$
t^{4} \mathcal{F}\left(\gamma \gg \gamma_{\mathrm{d}}, t \gg t_{\mathrm{ini}}\right) \simeq C \gamma^{2 \chi-3} .
$$

This expression matches with Eq. (11) provided that

$$
C=C_{\circ}(1-\nu)^{3-p}, \quad \chi=1-\frac{p}{2} .
$$

As discussed in the introduction, the PR functional form of the loop production function therefore yields a scaling loop distribution which matches with the one observed in the numerical simulations. Fixing the value of the above coefficients to those given in Eq. (2) can be considered as a renormalisation procedure to include the additional sources of loop formation, such as fragmentation and reconnection, not considered in the genuine analytical PR model.

In order to gain some intuition about the solution given in Eq. (33), we also determine its asymptotic forms in the two following limits. On the one hand, at length scales smaller than $\gamma_{\mathrm{d}}$, but larger than $\gamma_{\mathrm{c}}$ we recover the result of Ref. [59],

$$
t^{4} \mathcal{F}\left(\gamma_{\mathrm{c}}<\gamma \ll \gamma_{\mathrm{d}}, t \gg t_{\text {ini }}\right) \simeq \frac{C \mu}{2-2 \chi} \frac{\gamma^{2 \chi-2}}{\gamma_{\mathrm{d}}} .
$$

Hence, in this domain the loop number density distribution is in scaling but with a power law exponent reduced by one. On the other hand, for loops smaller than the gravitational backreaction scale $\gamma_{\mathrm{c}}$, we obtain for $\chi_{\mathrm{c}}>1$ that

$$
\begin{aligned}
t^{4} \mathcal{F}\left(\gamma \ll \gamma_{\mathrm{c}}, t \gg t_{\text {ini }}\right) \simeq & K\left(\frac{\gamma_{\mathrm{c}}+\gamma_{\mathrm{d}}}{\gamma_{\mathrm{d}}}\right)^{4-3 \nu} \\
& +C_{\mathrm{c}} \gamma_{\mathrm{d}}^{2 \chi_{\mathrm{c}}-3} \frac{\Gamma\left(\mu_{\mathrm{c}}+1\right) \Gamma\left(2 \chi_{\mathrm{c}}-2\right)}{\Gamma\left(2 \chi_{\mathrm{c}}-2+\mu_{\mathrm{c}}\right)},
\end{aligned}
$$

which does no longer depend on $\gamma$. As a result, the loop number distribution under the gravitational backreaction scale also reaches a scaling regime and even becomes scale independent. Let us further mention the extreme case $\chi_{\mathrm{c}}=1$, which, as previously 
discussed, yields a logarithmic divergence (in the radiation era, the matter epoch will be discussed in the next section),

$$
t^{4} \mathcal{F}\left(\gamma \ll \gamma_{\mathrm{c}}, t \gg t_{\text {ini }}\right) \simeq \frac{C_{\mathrm{c}} \mu_{\mathrm{c}}}{\gamma_{\mathrm{d}}} \ln \left(\frac{\gamma_{\mathrm{d}}}{\gamma}\right) .
$$

The constant value of Eq. (39) can be further expanded in the limit $\gamma_{\mathrm{c}} \ll \gamma_{\mathrm{d}}$. Using Eqs. (25) and (29), one finally finds that

$$
t^{4} \mathcal{F}\left(\gamma \ll \gamma_{\mathrm{c}} \ll \gamma_{\mathrm{d}}, t \gg t_{\text {ini }}\right) \simeq \frac{C \mu}{2-2 \chi} \frac{\gamma_{\mathrm{c}}^{2 \chi-2}}{\gamma_{\mathrm{d}}},
$$

which does no longer depend on $\chi_{\mathrm{c}}$. Note that Eq. (41) smoothly connects onto Eq. (38) at $\gamma=\gamma_{\mathrm{c}}$, as it is to be expected. The loop number density distribution is sensitive only to the scale at which gravitational backreaction occurs (at $\gamma_{c}$ ), but not how it occurs. This is fortunate since the precise form of the loop production function below the backreaction scale $\gamma_{\mathrm{c}}$ in unknown. For cosmological purposes, the only quantity that one needs to determine is thus $\gamma_{c}$. By comparing Eqs. (38) and (41), the physical interpretation is clear: the loop production function at $\gamma<\gamma_{\mathrm{c}}$ is always much smaller than for $\gamma>\gamma_{\mathrm{c}}$ (apart in the extreme case $\chi_{\mathrm{c}}=1$ ). As a result, most loops of size $\gamma<\gamma_{\mathrm{c}}$ at any given time were originally produced with a $\gamma>\gamma_{\mathrm{c}}$ and then shrunk down into the $\gamma<\gamma_{\mathrm{c}}$ domain by gravitational radiation. When $\gamma_{\mathrm{c}}$ is not much smaller than $\gamma_{\mathrm{d}}$, then $\chi_{\mathrm{c}}$ encodes how smooth the change of behaviour from Eq. (38) to Eq. (41) is, but still does not affect the value of $t^{4} \mathcal{F}(\gamma=0, t)$ significantly (see Fig. 2).

In Fig. 2, we have plotted $t^{4} \mathcal{F}(\gamma, t)$ in the radiation era and in the limit $t \gg t_{\text {ini }}$, i.e. when neglecting all terms in Eq. (33) involving a dependence on the initial time $t_{\text {ini. }}$. One recovers the three above mentioned domains with their power law behaviours of Eqs. (36), (38) and (41). Note that the overall shape does no longer depend on cosmic time $t$ but is universal: the loop distribution function is in its so-called "full scaling"

regime. The black curve has been obtained with $\chi_{\mathrm{c}}=2$ whereas the dashed gray curves correspond, from bottom to top, to $\chi_{\mathrm{c}}=4, \chi_{\mathrm{c}}=1.5$ and $\chi_{\mathrm{c}}=1$, respectively. As expected from Eq. (41), apart the logarithmic divergence when $\chi_{\mathrm{c}}=1$, deviations due to the detailed shape of the loop production function below the gravitational backreaction scale remain negligible.

Similar limits can be derived in the matter era by choosing $C$ and $\chi$ such that they match the relevant numbers of Eq. (2). The resulting loop distribution has the same overall shape as Fig. 2, with however different values for the power law indices. Notice also that the values of $\gamma_{c}$ in the radiation and matter era do not need to be the same.

In the next Section, we use the full solution given in Eq. (33) to discuss how the loop number density distribution relaxes towards its attractors in both the radiation and the matter era.

\section{Relaxation towards scaling}

In this Section, we first discuss the relaxation of the loop number density from a given initial distribution $\mathcal{N}_{\text {ini }}(\ell)$ in the radiation era. The motivation for these calculations 


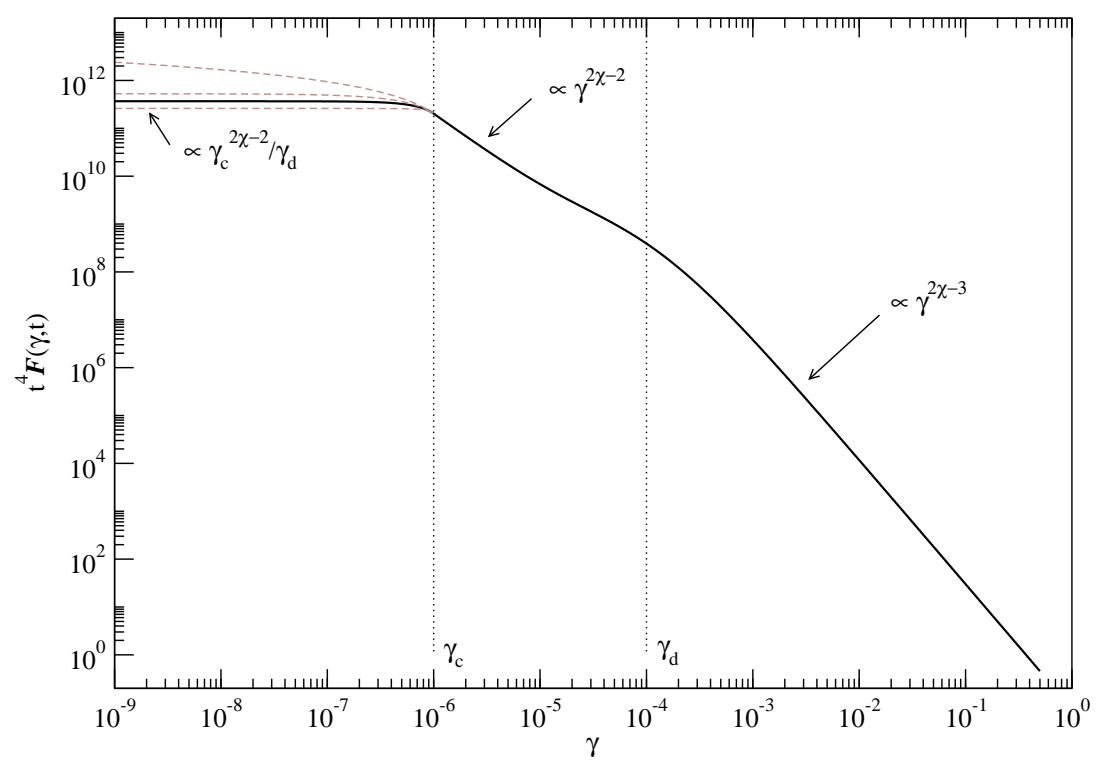

Figure 2. The loop number density distribution $t^{4} \mathcal{F}(\gamma, t)$ in the full scaling regime, i.e. when all terms involving $t_{\text {ini }}$ in Eq. (33) can be neglected. The solution has been plotted for the radiation era $(\nu=1 / 2)$ with $\chi$ and $C$ given by Eq. (37) and $\chi_{\mathrm{c}}=2$. The decay and gravitational backreaction scales have been fixed to the arbitrary values $\gamma_{\mathrm{d}}=10^{-4}$ and $\gamma_{\mathrm{c}}=10^{-6}$, respectively. The effects of changing $\chi_{\mathrm{c}}$ are illustrated by the dashed deviations which appear only for $\gamma<\gamma_{\mathrm{c}}$. From bottom to top, they correspond to the choices $\chi_{\mathrm{c}}=4, \chi_{\mathrm{c}}=1.5$ and the logarithmic divergent case $\chi_{\mathrm{c}}=1$.

comes from the fact that just after the string formation time, the loop number density is expected to be different from its cosmological attractor. Therefore, we want to determine how long it takes to relax towards the universal form of Fig. 2, As we show below, for any reasonable initial distribution and string formation temperature, $\mathcal{F}(\gamma, t)$ is expected to be in full scaling well before BBN. The second problem concerns the relaxation of the radiation scaling loop distribution towards the matter era attractor. Put it in a different way, the question that one may ask is whether we can still observe traces of loops formed in the radiation era in the matter loop distribution. Our objective here is to express our results directly in units of interest in our observable Universe. For this reason, we express the cosmic time $t$ in terms of the redshift $z$ according to Eqs. (4) and (5). We moreover use the currently favoured values of the cosmological parameters $h=0.72, \Omega_{\mathrm{m}_{0}} h^{2}=0.13$ and $\Omega_{\mathrm{r}_{0}} h^{2}=2.471 \times 10^{-5}[60$, and all lengths and times are expressed in units of Mpc unless specified otherwise. Note that for these cosmological parameters we find from the definition of $z_{*}$ in Eq. (6) that $z_{*} \simeq 3059$. 


\subsection{Radiation era}

As a well-motivated example, let us assume that the initial loop number density distribution is given by the Vachaspati-Vilenkin (VV) random walk model of Ref. [65],

$$
t_{\text {ini }}^{4} \mathcal{N}_{\text {ini }}(\ell)=C_{\text {i }}\left(\frac{t_{\text {ini }}}{\ell}\right)^{5 / 2} .
$$

Here, the constant $C_{\mathrm{i}}$ depends on the initial correlation length $\xi$ of the string network, an order of magnitude estimate being given by [65]

$$
C_{\mathrm{i}} \simeq\left(\frac{t_{\text {ini }}}{\xi}\right)^{3 / 2} .
$$

From Kibble's argument, causality imposes that $\xi / d_{\mathrm{h}}\left(t_{\text {ini }}\right) \leq 1$ and $C_{\mathrm{i}} \geq 1$. A maximum value for $C_{\mathrm{i}}$ can be estimated by imposing that the energy density of the string network at formation saturates the radiation energy density, i.e.

$$
H^{2}\left(t_{\text {ini }}\right) \simeq \frac{8 \pi G}{3} \frac{2 U}{\xi^{2}} .
$$

Neglecting constant coefficients, one then gets

$$
\left(\frac{\xi}{t_{\text {ini }}}\right)^{2} \simeq G U
$$

such that

$$
1 \leq C_{\mathrm{i}} \leq(G U)^{-3 / 4} .
$$

The loop distribution of Eq. (42) makes sense only for $\ell \geq \xi$, otherwise no loop can be produced. Also $\ell \leq d_{\mathrm{h} \mid \text { ini }}$, with $d_{\mathrm{h} \mid \text { ini }}=2 t_{\text {ini }}$ in the radiation era (since the long strings are already included in the loop production function, see the discussion above), and $\mathcal{N}_{\text {ini }}\left(\ell>2 t_{\text {ini }}\right)=0$. The maximum value of $t^{4} \mathcal{N}_{\text {ini }}$ then occurs for $\ell=\xi$ and can be estimated by

$$
t_{\mathrm{ini}}^{4} \mathcal{N}_{\text {ini }}(\xi) \simeq C_{\mathrm{i}}^{8 / 3} \leq(G U)^{-2} .
$$

By examining Eq. (33), let us first remark that since $\mathcal{N}_{\text {ini }}$ is maximal at a fixed physical length $\ell=\xi$, any effect associated with the initial loop distribution should be peaked at a non-constant $\gamma_{\xi}(t)=\xi / t$ and then travel towards smaller and smaller $\gamma$ values. This is what is observed in numerical simulations during relaxation of the initial conditions towards the loop scaling regime [41]. The above derivation does not consider the damped evolution epoch that is supposed to take place just after the phase transition responsible for string formation [3]. During this period, the background radiation is expected to smooth strings and loops such that some of the small loops should disappear. The above estimate should therefore be viewed as a upper bound for $\mathcal{N}_{\text {ini }}$.

Using the VV initial distribution of Eq. (42), it is immediate to check that, at fixed $\gamma$, the time dependence of all terms involving $\mathcal{N}_{\text {ini }}$ in Eq. (33) cancels. In other words, the initial loop density distribution remains, during some amount of time, on an equal footing with the freshly produced loops. However, since $\mathcal{N}_{\text {ini }}\left(\ell>2 t_{\text {ini }}\right)=0$, this regime 
can only be transient since the vanishing initial distribution at super-horizon loop sizes will "sweep leftwards" towards smaller $\gamma$ as time goes on. For any fixed value of $\gamma$, all terms involving $\mathcal{N}_{\text {ini }}$ in Eq. (33) vanish after a time $t_{\mathrm{h}}$ given by

$$
\frac{t_{\mathrm{h}}(\gamma)}{t_{\mathrm{ini}}}=\frac{2+\gamma_{\mathrm{d}}}{\gamma+\gamma_{\mathrm{d}}} .
$$

The relaxation time $t_{\mathrm{h}} / t_{\mathrm{ini}}$ is therefore a function of the scale of interest $\gamma$. For $\gamma \gg \gamma_{\mathrm{d}}$ (or $\gamma_{\mathrm{d}}=0$ ), it varies as $2 / \gamma$ such that it takes more time for the smaller loops to reach the scaling attractor, another property observed in the numerical simulations of Ref. [41]. For the smallest values $\gamma \ll \gamma_{\mathrm{d}}$, the relaxation time saturates to $t_{\mathrm{h}} / t_{\mathrm{ini}} \simeq 2 / \gamma_{\mathrm{d}}$, which is completely determined by the gravitational decay length scale. In terms of redshift, Eq. (48) reads

$$
\frac{1+z_{\mathrm{h}}(\gamma)}{1+z_{\text {ini }}}=\sqrt{\frac{\gamma+\gamma_{\mathrm{d}}}{2+\gamma_{\mathrm{d}}}},
$$

which is limited from below by

$$
\frac{z_{\mathrm{h}}}{z_{\text {ini }}}>\sqrt{\gamma_{\mathrm{d}} / 2} \text {. }
$$

Since $\gamma_{\mathrm{d}}$ can be small, it may take quite a long redshift range for the initial loop distribution to vanish and the observability of such transient effects also depends on $z_{\text {ini }}$.

If one assumes that the correlation length $\xi$ is approximately $\xi \simeq 1 / \sqrt{U}$, then Eq. (46) implies that $m_{\mathrm{Pl}} t_{\text {ini }}$ can vary from $1 / \sqrt{G U}$ to $1 /(G U)$, with $m_{\mathrm{Pl}}$ denoting the Planck mass. Taking the latter value and using Eq. (4) gives the lower bound

$$
z_{\text {ini }}>\frac{1}{\Omega_{\mathrm{r}_{0}}^{1 / 4}} \sqrt{\frac{m_{\mathrm{Pl}}}{H_{0}}} \sqrt{G U} \simeq 10^{31} \sqrt{G U} .
$$

Combined with Eqs. (9) and (50), one gets

$$
z_{\mathrm{h}}>\frac{G U}{\Omega_{\mathrm{r}_{0}}^{1 / 4}} \sqrt{\frac{\Gamma m_{\mathrm{Pl}}}{H_{0}}} \gtrsim 10^{32} G U .
$$

For string tensions of current cosmological interest, $G U \gtrsim 10^{-10}$ such that $z_{\mathrm{h}} \gtrsim 10^{22}$ : the decay of the initial loops is over well before BBN $\left(z_{\text {nuc }} \simeq 10^{10}\right)$. In fact, for all string tensions such that $G U>10^{-28}(\sqrt{U}>100 \mathrm{TeV})$, it is safe to claim that any initial loop remnants have been radiated away at the time of equality between radiation and matter $\left(z_{\text {eq }} \approx 10^{4}\right)$. Using the lowest value $t_{\text {ini }}=\xi$, the same statement would apply for strings as light as $G U \simeq 10^{-37}$, i.e. $\sqrt{U} \simeq 1 \mathrm{GeV}$.

We have just shown that all terms involving $\mathcal{N}_{\text {ini }}$ in Eq. (33) can be reasonably neglected at all redshifts $z<z_{\mathrm{h}}$. All the other terms involving $t_{\text {ini }}$ exhibit a power law decrease, up to a correction factor by the hypergeometric function which rapidly equals unity. In the radiation era, the biggest terms in Eq. (33) are those appearing in the expression for $\gamma>\gamma_{\mathrm{c}}$ and evolve as

$$
\left(\frac{t}{t_{\mathrm{ini}}}\right)^{2 \chi+1}\left(\frac{a_{\mathrm{ini}}}{a}\right)^{3} \simeq\left(\frac{z}{z_{\mathrm{ini}}}\right)^{1-4 \chi} .
$$




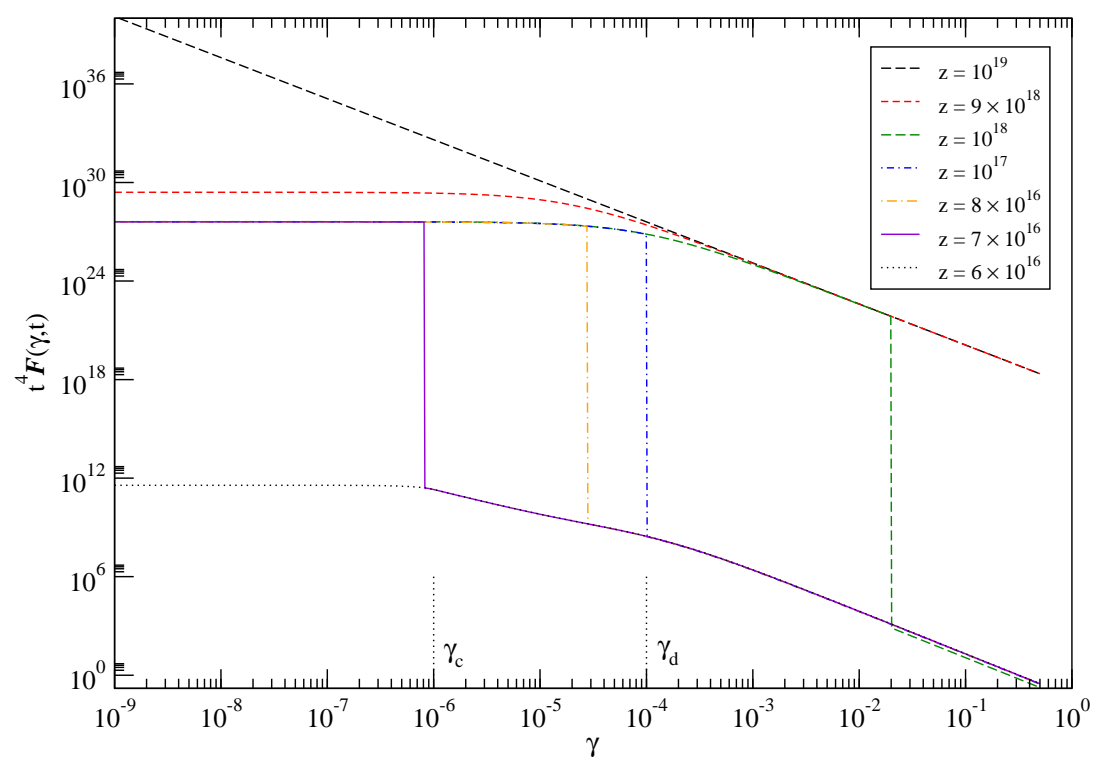

Figure 3. Relaxation towards scaling of the loop number density in the radiation era from a Vachaspati-Vilenkin configuration at $z_{\text {ini }}=10^{19}$. The step, whose position sweeps leftwards over the entire range of $\gamma$ with decreasing redshift, corresponds to the distance to the horizon at the string formation time above which $\mathcal{N}_{\text {ini }}=0$. It disappears after a finite time given by Eq. (48) such that it is no longer visible at $z=6 \times 10^{16}$ and the loop number density distribution is on the cosmological attractor.

Using the value $1-4 \chi \simeq 0.2$ from Eq. (2), they contribute less than $10 \%$ as soon as $z / z_{\text {ini }}<10^{-5}$, i.e. for

$$
z<z_{\%} \equiv 10^{26} \sqrt{G U}
$$

using Eq. (51). Again, for all relevant values of $G U$, these terms cancel well before BBN.

We have plotted in Fig. 3 the numerical solution stemming from Eq. (33) for an initial loop distribution given by Eq. (42). For illustrative purposes, we have chosen

$G U \simeq 10^{-14}$ such that the string formation redshift is at least $z_{\text {ini }} \simeq 10^{19}$ while letting the gravitational effect at the same value as before. The relaxation towards scaling is completed at $z \simeq 10^{16}$, as one expects from Eq. (54).

\subsection{Matter era}

It follows from the previous discussion that the cosmic string loop number density distribution expected at the end of the radiation era is given by its scaling solution described by the power laws of Eqs. (36), (38) and (41). Taking this shape as the initial distribution $\mathcal{N}_{*}$ at $z=z_{*}$ [see Eq. (6)] for the subsequent evolution in the matter era, we can now discuss how the loop number density relaxes towards its new scaling solution in the matter era.

For $\nu=2 / 3$, it follows from Eq. (23) that $\mu=1-2 \chi$ and the hypergeometric 
function in Eqs. (24) simplifies to the polynomial expression [66]

$$
f(x)=\frac{1}{(1-x)^{2-2 \chi}}\left(1-\frac{x}{2-2 \chi}\right),
$$

and similarly for $f_{\mathrm{c}}(x)$, provided $\chi_{\mathrm{c}} \neq 1$. In the matter era, the extreme case $\chi_{\mathrm{c}}=1$ would produce a logarithmic divergence in time for $\gamma<\gamma_{\mathrm{c}}$ suggesting that it is no longer physically acceptable. Therefore, this extreme case will not be considered in the following. Concerning the values of $\gamma_{\mathrm{d}}$ and $\gamma_{\mathrm{c}}$, one does not expect the former to change significantly from the radiation to the matter era [as one can see from Eq. (9)] ]. However, given that $\gamma_{\mathrm{c}}$ is closely related to the tangent correlator [see Eq. (14)], it assumes a lower value in the matter dominated epoch.

As for the radiation era, it suffices to discuss the relaxation of the loop number density distribution in the regime $\gamma>\gamma_{\mathrm{c}}$ since the smaller structures relax even faster. We denote by $\chi_{\mathrm{R}}, C_{\mathrm{R}}$ and $\chi_{\mathrm{M}}, C_{\mathrm{M}}$ the respective values of $\chi$ and $C$ in the radiation and matter era. The time dependence associated with the terms involving $\mathcal{N}_{*}$ (the matter era equivalent of $\mathcal{N}_{\text {ini }}$ for the radiation epoch) in Eq. (33) shows that, unlike when starting from the VV distribution, they are now damped during matter domination and become sub-dominant compared to the scaling solution as soon as $z<z_{\mathrm{d}}(\gamma)$, with

$$
\frac{1+z_{\mathrm{d}}(\gamma)}{1+z_{*}}=\left(\frac{C_{\mathrm{M}}}{C_{\mathrm{R}}}\right)^{1 /\left(3 / 2-3 \chi_{\mathrm{R}}\right)} \gamma^{2\left(\chi_{\mathrm{M}}-\chi_{\mathrm{R}}\right) /\left(3 / 2-3 \chi_{\mathrm{R}}\right)} .
$$

This expression was derived assuming $\gamma \gg \gamma_{\mathrm{d}}$. Using the numerical values of Eq. (2), this corresponds to

$$
\frac{1+z_{\mathrm{d}}(\gamma)}{1+z_{*}} \simeq 0.16 \gamma^{0.2}
$$

The damping terms hence are more efficient for the larger length scales and the scaling solution takes over after an expansion factor of typically $\mathcal{O}(10)$, i.e. when $z_{\mathrm{d}} \simeq 300$. For $\gamma_{\mathrm{c}}<\gamma \ll \gamma_{\mathrm{d}}$, one finds the same expression as in Eq. (57) up to the replacement $\gamma \rightarrow \gamma_{\mathrm{d}}$. As a result, if $\gamma_{\mathrm{d}}$ is very small, the scaling solution for the small loops may not overcome $\mathcal{N}_{*}$ before today. For instance, if $\gamma \leq \gamma_{\mathrm{d}}=10^{-14}$, one finds $z_{\mathrm{d}} \simeq 0$. Physically, it means that some of the small loops today may actually come from the radiation era. Notice that, in addition to the damping effect, there is the horizon cutoff $\mathcal{N}_{*}\left(\ell>2 t_{*}\right)=0$. This imposes that all terms involving $\mathcal{N}_{*}$ exactly vanish for $z<z_{\mathrm{h}}(\gamma)$ with

$$
\frac{1+z_{\mathrm{h}}(\gamma)}{1+z_{*}}=\left(\frac{\gamma+\gamma_{\mathrm{d}}}{2+\gamma_{\mathrm{d}}}\right)^{2 / 3} \text {. }
$$

However, compared to Eq. (57), $z_{\mathrm{h}}<z_{\mathrm{d}}$ such that the horizon cutoff is less efficient than damping. Let us remark that for the typical value of $G U \simeq 10^{-7}$, Eq. (9) implies that $\gamma_{\mathrm{d}} \simeq 10^{-5}$ and the residual loops from the radiation era disappear around $z_{\mathrm{h}} \simeq 30$. Finally, the second term involving a dependence on $t_{*}$, the initial time for the matter epoch, in Eq. (33) evolves as

$$
\left(\frac{t}{t_{*}}\right)^{2 \chi_{\mathrm{M}}+1}\left(\frac{a_{*}}{a}\right)^{3} \simeq\left(\frac{1+z}{1+z_{*}}\right)^{3 / 2-3 \chi_{\mathrm{M}}},
$$




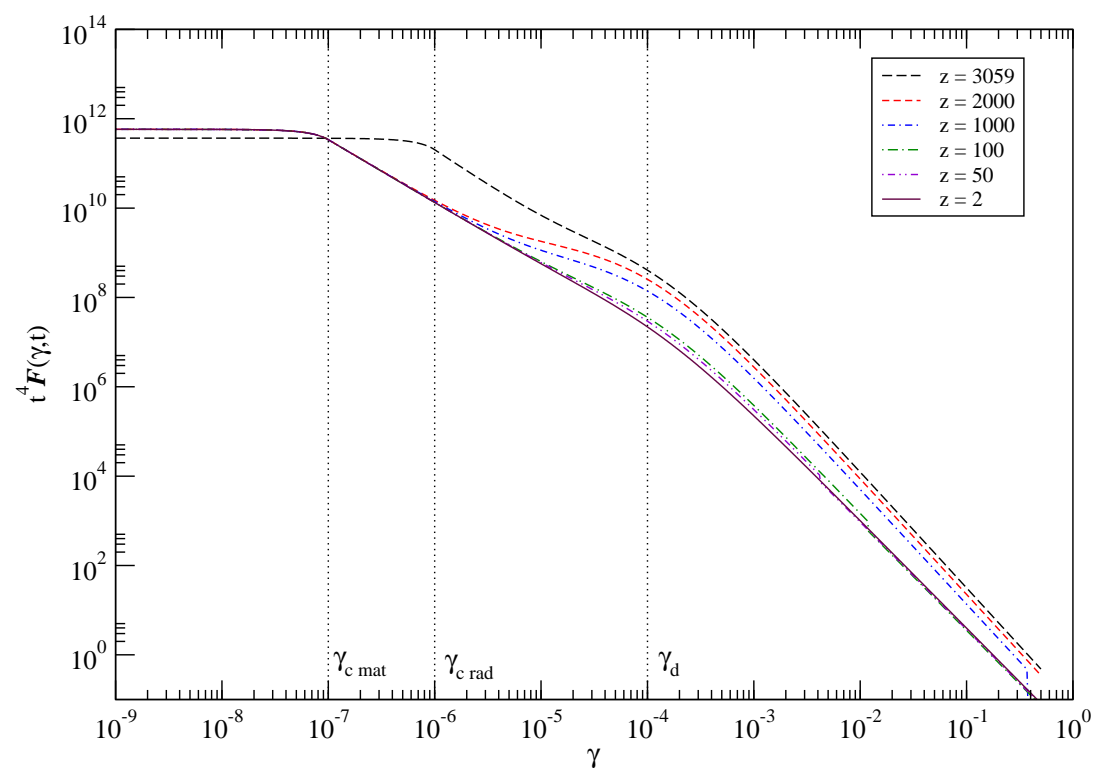

Figure 4. Relaxation of the loop number density distribution towards the matter era scaling from the radiation era scaling solution at $z=z_{*}$. We have chosen the arbitrary values $\gamma_{\mathrm{d}}=10^{-4}, \gamma_{\mathrm{c} \mid \mathrm{rad}}=10^{-6}$ and $\gamma_{\mathrm{c} \mid \text { mat }}=10^{-7}$ while the other constants have been fixed to match numerical simulations [see Eq. (2)]. The relaxation takes longer for $\gamma \simeq \gamma_{\mathrm{d}}$ and is completed at $z \simeq 70$. Lower values of $\gamma_{\mathrm{d}}$ would increase the relaxation time according to Eq. (57) such that some small residual loops may still be present today.

and contributes less than $10 \%$ as soon as $z<z_{\%} \simeq 65$, independently of the value of $\gamma_{\mathrm{d}}$. In Fig. 4, we have plotted the loop distribution in the matter era when $\mathcal{N}_{*}$ is given by the radiation era scaling solution. As expected from the above discussion, we find the relaxation time to be longer for loops with size $\gamma_{\mathrm{c}}<\gamma \lesssim \gamma_{\mathrm{d}}$ while the redshift at which the matter scaling solution dominates is compatible with Eq. (57).

To conclude this section, the loop number density distribution today is expected to be in full scaling on all length scales provided $\gamma_{\mathrm{d}}>10^{-14}$. If $\gamma_{\mathrm{d}}<10^{-14}$, then some of the loops having a size $\gamma_{\mathrm{c}}<\gamma \lesssim \gamma_{\mathrm{d}}$ have been formed in the radiation era and the distribution is accordingly modified. On the other length scales, the distribution matches with the matter era attractor. For those cases, one can use the full expression given in Eq. (33) to get the precise loop number density distribution, as plotted in Fig. 4.

\subsection{Cosmological applications}

3.3.1. Density parameter of cosmic string loops We may now use our knowledge of the loop number density distribution in the matter era to compute various quantities describing the cosmological impact of these loops today. For simplicity, we neglect the recent change in the universal expansion from matter to vacuum energy domination and continue to use Eq. (5) to relate cosmic time to the redshift. Furthermore, we assume that $\gamma_{\mathrm{d}}>10^{-14}$ such that the distribution is in its (matter era) full scaling regime. From 
Eqs. (33) and (55), the loop number density distribution then simplifies to

$$
\begin{aligned}
& t^{4} \mathcal{F}\left(\gamma \geq \gamma_{\mathrm{c}}, t\right)=\frac{C}{\gamma^{2-2 \chi}\left(\gamma+\gamma_{\mathrm{d}}\right)}\left(1-\frac{1}{2-2 \chi} \frac{\gamma_{\mathrm{d}}}{\gamma+\gamma_{\mathrm{d}}}\right), \\
& t^{4} \mathcal{F}\left(\gamma<\gamma_{\mathrm{c}}, t\right)=\frac{C_{\mathrm{c}} \gamma^{2 \chi_{\mathrm{c}}-2}}{\gamma+\gamma_{\mathrm{d}}}\left(1+\frac{1}{2 \chi_{\mathrm{c}}-2} \frac{\gamma_{\mathrm{d}}}{\gamma+\gamma_{\mathrm{d}}}\right)+K\left(\frac{\gamma_{\mathrm{c}}+\gamma_{\mathrm{d}}}{\gamma+\gamma_{\mathrm{d}}}\right)^{2},
\end{aligned}
$$

where $C_{\mathrm{c}}$ and $K$ are explicitly given in Eqs. (25) and (29) by using Eq. (55), and we no longer distinguish between $\chi_{\mathrm{R}}$ and $\chi_{\mathrm{M}}$ as only the latter is relevant for the following considerations. The energy density in the form of cosmic string loops of all sizes is given by

$$
\rho_{\circ}=\frac{U}{t^{2}} \int_{0}^{3} t^{4} \mathcal{F}(\gamma, t) \gamma \mathrm{d} \gamma,
$$

where the upper value comes from the distance to the horizon in the matter era using $\nu=2 / 3$ (note that this expression does not include the Kibble's loops whose contribution remains, however, negligible). Although Eq. (61) can be explicitly integrated in terms of hypergeometric functions, the resulting expression is not particularly illuminating. A very good approximation can be obtained by piecewise integrating the asymptotic power law expansions of Eqs. (36), (38) and (41) in the three domains $\gamma \leq \gamma_{\mathrm{c}}, \gamma_{\mathrm{c}}<\gamma \leq \gamma_{\mathrm{d}}$, and $\gamma>\gamma_{\mathrm{d}}$. After some algebra, one finds

$$
\begin{aligned}
\frac{\rho_{\mathrm{o}} t^{2}}{U} \simeq & \frac{C}{\gamma_{\mathrm{d}}^{1-2 \chi}}\left[\frac{1}{4 \chi(1-\chi)(1-2 \chi)}-\frac{1-2 \chi}{4 \chi}\left(\frac{\gamma_{\mathrm{c}}}{\gamma_{\mathrm{d}}}\right)^{2 \chi}\right. \\
& \left.-\frac{1}{1-2 \chi}\left(\frac{\gamma_{\mathrm{d}}}{3}\right)^{1-2 \chi}\right],
\end{aligned}
$$

which can be further approximated for $\gamma_{\mathrm{c}} \ll \gamma_{\mathrm{d}} \ll 1$ by

$$
\frac{\rho_{\circ} t^{2}}{U} \simeq \frac{C}{4 \chi(1-\chi)(1-2 \chi)} \frac{1}{\gamma_{\mathrm{d}}^{1-2 \chi}} .
$$

This expression clearly shows that the energy density in the form of loops is essentially fixed by the gravitational decay scale $\gamma_{\mathrm{d}}$ and the scaling function properties through $C$ and $\chi$. Smaller $\gamma_{\mathrm{d}}$ implies larger values for $\rho_{\mathrm{o}} / U$. Notice also that the scaling evolution is similar to that of the long string ones; the quantity $t^{2} \rho_{\circ} / U$ is time independent and universal. Doing the integration exactly and then expanding the hypergeometric functions gives the same result, up to the first numerical coefficient which changes by fifteen percents and reads $C \pi /[(2-2 \chi) \sin (2 \pi \chi)]$. In terms of the density parameter $\Omega_{\circ} \equiv \rho_{\circ} / \rho_{\text {crit }}$, where $\rho_{\text {crit }}$ is the critical density for a flat universe today, we find

$$
\Omega_{\circ}=\frac{3 \pi^{2} C}{(1-\chi) \sin (2 \pi \chi)} \frac{G U}{\gamma_{d}^{1-2 \chi}},
$$

which is valid at all scaling times during the matter era. Up to some small additional dilution factor coming from the recent cosmological constant domination, this expression may be extrapolated until today. Using Eq. (9) with $\Gamma \simeq 10^{2}$ and the numbers derived before, one finds

$$
\Omega_{\circ} \simeq 0.10 \times(G U)^{0.59} .
$$


This cannot exceed $10^{-5}$ with the values of $G U$ currently allowed by CMB observations, i.e. $G U \leq 7 \times 10^{-7}$, and hence Nambu-Goto cosmic string loops cannot provide a viable Dark Matter candidate, a scenario which has been dicussed in Ref. [67].

3.3.2. Number density of loops in a box of size L Again assuming the full scaling solution in the matter era, we can estimate the number density of cosmic string loops present around us today. Taking a box of size $L$, Eq. (60) can be used to determine the number density $n_{L}$ of loops having a length $\ell \leq L$ at $t$ :

$$
t^{3} n_{L}=\int_{0}^{L / t} t^{4} \mathcal{F}(\gamma, t) \mathrm{d} \gamma
$$

Assuming the box is big enough, with $\gamma_{\mathrm{c}}<\gamma_{\mathrm{d}} \ll L / t$, we find the dominant part of the loop density to be independent of $L$,

$$
t^{3} n_{L}=\frac{2 \chi_{\mathrm{c}}-2 \chi}{2 \chi_{\mathrm{c}}-1} \frac{C}{\gamma_{\mathrm{d}} \gamma_{\mathrm{c}}^{1-2 \chi}} .
$$

As expected, the dependence in $\chi_{\mathrm{c}}$ is very weak such that the first term in the previous equation can be approximated to unity. Using Eqs. (9) and (14) with $\Gamma \simeq 10^{2}$ as before and $\Upsilon \simeq 10$, yields

$$
n_{L} \simeq \frac{6.1 \times 10^{-5}}{t^{3}}(G U)^{-1.65} .
$$

Using $t=t_{0}$ from Eq. (5) evaluated for $z=0$ (which again amounts to neglecting the recent change to a vacuum energy dominated universe) with $G U \simeq 7 \times 10^{-7}$ gives the lower bound

$$
n_{L} \simeq 5.5 \times 10^{-6} \mathrm{Mpc}^{-3} .
$$

This number is quite small, but interestingly it scales as a negative power of $G U$, such that loops from very light cosmic strings are the more numerous. Our result suggests that lensing events [68] from loops are more frequent for low values of $G U$ (but also of smaller amplitude). Note that with the above string tension, the gravitational backreaction length is at $\gamma_{\mathrm{c}} t_{0} \simeq 8 \mathrm{pc}$, and the gravitational decay length at $\gamma_{\mathrm{d}} t_{0} \simeq 380 \mathrm{kpc}$.

\section{Conclusions}

In this paper, we have proposed a phenomenological model of Nambu-Goto cosmic string loop evolution based on the Polchinski-Rocha loop production function, which we adjusted to fit numerical simulations. We have considered both loop decay by gravitational wave emission and string smoothing due to gravitational backreaction. We have found that the loop number density distribution rapidly assumes a universal form on all length scales, which ends up being insensitive to the details of the backreaction effects. It solely depends on the scale $\gamma_{\mathrm{c}}$ below which these effects dominate. In the matter era, the energy density parameter of loops $\Omega_{\circ}$ is a positive power of the string tension $G U$ and ends up being completely negligible. Hence, cosmic string loops are 
unfit to account for the Dark Matter contribution to our Universe's energy density. On the other hand, the density number of loops is boosted by a negative power of $G U$, meaning that very low tension loops could actually be abundant in the present Universe.

Our work may be extended along several lines. For instance, since the universal loop distribution plotted in Fig. 2 is significantly different than the loop distributions used so far in the literature, various constraints on $G U$ coming from gravitational wave emission, lensing or pulsar-timing should be re-examined [32, 33, 69, 34, 70]. All along the calculation, we have kept $\gamma_{d}$ and $\gamma_{c}$ as undetermined parameters (apart from the numerical examples) since these parameters may equally well be used to represent other physical effects. For instance, $\gamma_{d}$ does not need to be given by Eq. (9) but could represent the decay rate of another energy loss mechanism, such as particle emission, provided it can still be described phenomenologically by Eq. (8) . Another extension would be the implementation of a low intercommutation probability (as in the PR model, we have set this probability $P=1$ throughout this work) to extend our approach to the evolution of cosmic superstring loops.

\section{Acknowledgments}

It is a pleasure to thank Patrick Peter and Daniele Steer for enlightening discussions. The work of L. L. and C. R. is partially supported by the Belgian Federal Office for Science, Technical and Cultural Affairs, under the Inter-university Attraction Pole Grant No. P6/11. The work of M. S. is partially supported by the Sciences \& Technology Facilities Council (STFC-UK), Particle Physics Division, under the grant ST/G000476/1 "Branes, Strings and Defects in Cosmology".

\section{References}

[1] T. W. B. Kibble, Topology of cosmic domains and strings., J. Phys. A 9 (1976) 1387-1398.

[2] M. B. Hindmarsh and T. W. B. Kibble, Cosmic strings, Rept. Prog. Phys. 58 (1995) 477-562, hep-ph/9411342.

[3] A. Vilenkin and E. P. S. Shellard, Cosmic Strings and Other Topological Defects. Cambridge University Press, Cambridge, England, July, 2000.

[4] M. Sakellariadou, Cosmic strings, Lect. Notes Phys. 718 (2007) 247-288, hep-th/0602276.

[5] D. Kirzhnits and A. Linde, Macroscopic consequences of the Weinberg model, Phys. Lett. B 42 (Dec., 1972) 471-474.

[6] R. Jeannerot, J. Rocher, and M. Sakellariadou, How generic is cosmic string formation in SUSY GUTs, Phys. Rev. D68 (2003) 103514, hep-ph/0308134.

[7] J. Rocher and M. Sakellariadou, D-term inflation, cosmic strings, and consistency with cosmic microwave background measurement, Phys. Rev. Lett. 94 (2005) 011303, hep-ph/0412143.

[8] C. P. Burgess et. al., The Inflationary Brane-Antibrane Universe, JHEP 07 (2001) 047, hep-th/0105204.

[9] S. Sarangi and S. H. H. Tye, Cosmic string production towards the end of brane inflation, Phys. Lett. B536 (2002) 185-192, hep-th/0204074.

[10] G. Dvali and A. Vilenkin, Formation and evolution of cosmic D-strings, JCAP 0403 (2004) 010, hep-th/0312007. 
[11] N. T. Jones, H. Stoica, and S. H. H. Tye, The production, spectrum and evolution of cosmic strings in brane inflation, Phys. Lett. B563 (2003) 6-14, hep-th/0303269.

[12] A.-C. Davis and T. Kibble, Fundamental cosmic strings, Contemp. Phys. 46 (Sept., 2005) 313-322, hep-th/0505050.

[13] M. Sakellariadou, Cosmic Superstrings, Phil. Trans. Roy. Soc. Lond. A366 (2008) 2881-2894, arXiv:0802.3379.

[14] E. J. Copeland and T. W. B. Kibble, Cosmic Strings and Superstrings, Proc. Roy. Soc. Lond. A466 (2010) 623-657, arXiv:0911.1345.

[15] M. Sakellariadou, Cosmic Strings and Cosmic Superstrings, Nucl. Phys. Proc. Suppl. 192-193 (2009) 68-90, arXiv:0902.0569.

[16] A. Albrecht and N. Turok, Evolution of cosmic string networks, Phys. Rev. D40 (Aug., 1989) 973-1001.

[17] D. P. Bennett and F. R. Bouchet, Cosmic-string evolution, Phys. Rev. Lett. 63 (Dec., 1989) $2776-2779$.

[18] D. P. Bennett and F. R. Bouchet, High-resolution simulations of cosmic-string evolution. I. Network evolution, Phys. Rev. D41 (Apr., 1990) 2408-2433.

[19] B. Allen and P. Shellard, Cosmic-string evolution - A numerical simulation, Phys. Rev. Lett. 64 (Jan., 1990) 119-122.

[20] N. Jarosik et. al., Seven-Year Wilkinson Microwave Anisotropy Probe (WMAP) Observations: Sky Maps, Systematic Errors, and Basic Results, arXiv:1001.4744

[21] N. Bevis, M. Hindmarsh, M. Kunz, and J. Urrestilla, Fitting CMB data with cosmic strings and inflation, Phys. Rev. Lett. 100 (2008) 021301, astro-ph/0702223.

[22] A. A. Fraisse, C. Ringeval, D. N. Spergel, and F. R. Bouchet, Small-Angle CMB Temperature Anisotropies Induced by Cosmic Strings, Phys. Rev. D78 (2008) 043535, arXiv:0708.1162.

[23] K. Takahashi et. al., Non-Gaussianity in Cosmic Microwave Background Temperature Fluctuations from Cosmic (Super-)Strings, JCAP 0910 (2009) 003, arXiv:0811.4698.

[24] M. Hindmarsh, C. Ringeval, and T. Suyama, The CMB temperature bispectrum induced by cosmic strings, Phys. Rev. D80 (2009) 083501, arXiv:0908.0432.

[25] M. Hindmarsh, C. Ringeval, and T. Suyama, The CMB temperature trispectrum of cosmic strings, Phys. Rev. D81 (2010) 063505, [arXiv:0911.1241].

[26] D. M. Regan and E. P. S. Shellard, Cosmic String Power Spectrum, Bispectrum and Trispectrum, Phys. Rev. D82 (2010) 063527, [arXiv:0911.2491].

[27] D. Yamauchi et. al., Skewness in CMB temperature fluctuations from curved cosmic (super-)strings, arXiv:1004.0600.

[28] M. Landriau and E. P. S. Shellard, Cosmic String Induced CMB Maps, arXiv:1004.2885

[29] N. Bevis, M. Hindmarsh, M. Kunz, and J. Urrestilla, CMB power spectra from cosmic strings: predictions for the Planck satellite and beyond, Phys. Rev. D82 (2010) 065004, arXiv:1005.2663.

[30] C. Ringeval, Cosmic strings and their induced non-Gaussianities in the cosmic microwave background, Adv. Astron. 2010 (2010) 380507, arXiv:1005.4842.

[31] B. Allen and E. P. S. Shellard, Gravitational radiation from cosmic strings, Phys. Rev. D 45 (Mar, 1992) 1898-1912.

[32] T. Damour and A. Vilenkin, Gravitational wave bursts from cusps and kinks on cosmic strings, Phys. Rev. D64 (2001) 064008, gr-qc/0104026.

[33] X. Siemens et. al., Gravitational wave bursts from cosmic (super)strings: Quantitative analysis and constraints, Phys. Rev. D73 (2006) 105001, gr-qc/0603115.

[34] S. Olmez, V. Mandic, and X. Siemens, Gravitational-Wave Stochastic Background from Kinks and Cusps on Cosmic Strings, arXiv:1004.0890.

[35] F. Dubath and J. V. Rocha, Periodic gravitational waves from small cosmic string loops, Phys. Rev. D76 (2007) 024001, gr-qc/0703109.

[36] G. Vincent, N. D. Antunes, and M. Hindmarsh, Numerical Simulations of String Networks in the 
Abelian-Higgs Model, Phys. Rev. Lett. 80 (Mar., 1998) 2277-2280, hep-ph/9708427.

[37] J. N. Moore, E. P. S. Shellard, and C. J. A. P. Martins, Evolution of Abelian-Higgs string networks, Phys. Rev. D65 (Jan., 2001) 023503, hep-ph/0107171.

[38] M. Hindmarsh, S. Stuckey, and N. Bevis, Abelian Higgs Cosmic Strings: Small Scale Structure and Loops, Phys. Rev. D79 (2009) 123504, arXiv:0812.1929.

[39] G. R. Vincent, M. Hindmarsh, and M. Sakellariadou, Scaling and small scale structure in cosmic string networks, Phys. Rev. D56 (1997) 637-646, astro-ph/9612135.

[40] V. Vanchurin, K. Olum, and A. Vilenkin, Cosmic string scaling in flat space, Phys. Rev. D72 (2005) 063514, gr-qc/0501040.

[41] C. Ringeval, M. Sakellariadou, and F. Bouchet, Cosmological evolution of cosmic string loops, JCAP 0702 (2007) 023, astro-ph/0511646.

[42] V. Vanchurin, K. D. Olum, and A. Vilenkin, Scaling of cosmic string loops, Phys. Rev. D74 (2006) 063527, gr-qc/0511159.

[43] C. J. A. P. Martins and E. P. S. Shellard, Fractal properties and small-scale structure of cosmic string networks, Phys. Rev. D73 (2006) 043515, astro-ph/0511792.

[44] K. D. Olum and V. Vanchurin, Cosmic string loops in the expanding universe, Phys. Rev. D75 (2007) 063521, astro-ph/0610419.

[45] A. Vilenkin, Gravitation radiation from cosmic strings., Phys. Lett. B 107 (1981) 47-50.

[46] M. Sakellariadou, Gravitational waves emitted from infinite strings, Phys. Rev. D42 (1990) 354-360.

[47] M. Hindmarsh, Gravitational radiation from kinky infinite strings, Phys. Lett. B251 (1990) $28-33$.

[48] X. Siemens and K. D. Olum, Gravitational radiation and the small-scale structure of cosmic strings, Nucl. Phys. B611 (2001) 125-145, gr-qc/0104085.

[49] X. Siemens, K. D. Olum, and A. Vilenkin, On the size of the smallest scales in cosmic string networks, Phys. Rev. D66 (2002) 043501, gr-qc/0203006.

[50] J. Polchinski and J. V. Rocha, Cosmic string structure at the gravitational radiation scale, Phys. Rev. D75 (2007) 123503, gr-qc/0702055.

[51] D. Austin, E. J. Copeland, and T. W. B. Kibble, Evolution of cosmic string configurations, Phys. Rev. D48 (1993) 5594-5627, hep-ph/9307325.

[52] C. J. A. P. Martins and E. P. S. Shellard, Quantitative String Evolution, Phys. Rev. D54 (1996) 2535-2556, hep-ph/9602271.

[53] E. J. Copeland, T. W. B. Kibble, and D. A. Steer, The evolution of a network of cosmic string loops, Phys. Rev. D58 (1998) 043508, hep-ph/9803414.

[54] C. J. A. P. Martins and E. P. S. Shellard, Extending the velocity-dependent one-scale string evolution model, Phys. Rev. D65 (2002) 043514, hep-ph/0003298.

[55] J. Polchinski and J. V. Rocha, Analytic study of small scale structure on cosmic strings, Phys. Rev. D74 (2006) 083504, hep-ph/0606205.

[56] F. Dubath, J. Polchinski, and J. V. Rocha, Cosmic String Loops, Large and Small, Phys. Rev. D77 (2008) 123528, arXiv:0711.0994.

[57] V. Vanchurin, Non-linear dynamics of cosmic strings with non-scaling loops, Phys. Rev. D82 (2010) 063503, arXiv:1005.1680.

[58] V. Vanchurin, Semi-scaling cosmic strings, arXiv:1005.5721.

[59] J. V. Rocha, Scaling solution for small cosmic string loops, Phys. Rev. Lett. 100 (2008) 071601, arXiv:0709.3284.

[60] E. Komatsu et. al., Seven-Year Wilkinson Microwave Anisotropy Probe (WMAP) Observations: Cosmological Interpretation, arXiv:1001.4538,

[61] E. J. Copeland and T. W. B. Kibble, Kinks and small-scale structure on cosmic strings, Phys. Rev. D80 (2009) 123523, arXiv:0909.1960.

[62] T. Vachaspati and A. Vilenkin, Formation and Evolution of Cosmic Strings, Phys. Rev. D30 (1984) 2036. 
[63] G. Karra and R. J. Rivers, Initial vortex densities after a temperature quench, Phys. Lett. B414 (1997) 28-33, hep-ph/9705243.

[64] M. Hindmarsh and A. Rajantie, Phase transition dynamics in the hot Abelian Higgs model, Phys. Rev. D64 (2001) 065016, hep-ph/0103311.

[65] T. Vachaspati and A. Vilenkin, Formation and evolution of cosmic strings, Phys. Rev. D30 (Nov., 1984) 2036-2045.

[66] I. S. Gradshteyn and I. M. Ryzhik, Table of Integrals, Series, and Products. Academic Press, New York and London, 1965.

[67] Y. Cui and D. E. Morrissey, Non-Thermal Dark Matter from Cosmic Strings, Phys. Rev. D79 (2009) 083532, arXiv:0805.1060.

[68] A. A. de Laix and T. Vachaspati, Gravitational lensing by cosmic string loops, Phys. Rev. D54 (1996) 4780-4791, astro-ph/9605171.

[69] M. S. Pshirkov and A. V. Tuntsov, Local constraints on cosmic string loops from photometry and pulsar timing, Phys. Rev. D81 (2010) 083519, arXiv:0911.4955.

[70] A. V. Tuntsov and M. S. Pshirkov, Quasar variability limits on cosmological density of cosmic strings, Phys. Rev. D81 (2010) 063523, arXiv:1001.4580. 